\section{ENVIRONMENTAL TOBACCO SMOKE EXPOSURE AND CHILD NEURODEVELOPMENT}

\section{K. Polanska}

Department of Environmental Epidemiology, Nofer Institute of Occupational Medicine, Lodz, Poland

Background: In Poland about $30 \%$ of children is exposed to environmental tobacco smoke during prenatal period and more than $50 \%$ in postnatal one. Such exposure has serious health consequences including negative effect on child neurodevelopment. The aim of the study was to assess the effect of environmental tobacco smoke (ETS) exposure on child psychomotor development.

Material and methods: The study population consisted of children with well assessed prenatal exposure to environmental tobacco smoke (three times analysis of cotinine level in saliva of pregnant women). Assessment of child ETS exposure within two years after birth was based on questionnaires conducted with mothers, confirmed by biochemical verification of cotinine level in child urine. The cotinine level in biological samples was analyzed using Liquid Chromatography (HPLC) with Tandem Mass Spectrometry (MSMS). The Bayley Scale for Infant and Toddler Development (BESID-III) was used for the evaluation of child neurodevelopment.

Results: Multivariative analysis (including gender, birth order of the child and parental educational status) indicated the statistically significant association between child ETS exposure and cognitive development $(b=-4,0 ; p=0,04)$. ETS exposure has also negative impact on motor $(b=$ $2.7 ; p=0.2)$ and language $(b=-3.4 ; p=0.08)$ abilities of the child although the results were not statistically significant.

Conclusions: Maternal smoking was found to be related to a decrease child neurodevelopment although it impossible to separate the prenatal from postnatal exposure. All effort should be taken to eliminate the child ETS exposure.

\section{WORK IN GREENHOUSES AND INFANTS' BIRTH WEIGH}

\section{J. Jurewicz}

\section{Environmental Epidemiology, Nofer Institute of Occupational Medicine, Lodz, Poland}

Problem about exposure to pesticides is important in greenhouses where work is performed in warm microclimate during the most time of the year, involves usually moderately intense or heavy work. The working conditions in greenhouses might involve indirect exposure to pesticides resulting from contact with pesticide-treated flowers and vegetables.

Methods: 460 women who had been working for a period of at least 2 years in greenhouses in Poland were asked to participate in the project. We classified pregnancies of women working in, and out of, greenhouses on the basis of energy expenditure during mother's work into three groups: light work (200-700 kcal/shift); moderate work (701$1000 \mathrm{kcal} / \mathrm{shift})$; and heavy work (1001-1200 kcal/ shift). Information about application of pesticides was received from persons responsible for chemical protection in each examined greenhouse. Based on the Pan American Pesticide Database we identified pesticides which were classified as reproductive and developmental (RD) toxins.

Results: The study among women working in greenhouse revealed that the mean birth weight of infants whose mothers worked in greenhouse during pregnancy (work expenditure > $1000 \mathrm{kcal} /$ shift) was $177 \mathrm{~g}$ lower than that of those whose mothers worked out of greenhouse (light work < $700 \mathrm{kcal} / \mathrm{shift})(p=0.05)$. Mothers who during work in greenhouse were potentially exposed to RD pesticides, delivered infants with birth weight lower by about $70 \mathrm{~g}$. than infants' mothers not working at places where pesticides RD were applied, but these findings were not statistically significant.

\section{7}

\section{PRENATAL AND POSTNATAL CHILD EXPOSURE TO ENVIRONMENTAL TOBACCO SMOKE}

\section{K. Polanska}

Department of Environmental Epidemiology, Nofer Institute of Occupational Medicine, Lodz, Poland

Background: Environmental tobacco smoke exposure (ETS) is associated with poor pregnancy 
outcome including increases risk of spontaneous abortion, stilbirth, low birth weight, preterm delivery and sudden infant death syndrome. ETS exposure after birth can increase the risk of sudden infant death syndrome, respiratory infections, asthma, middle ear diseases or even cancer and it can cause neurodevelopmental and behavioural problems in children.

The aim of the study was to evaluate the prenatal and postnatal child exposure to environmental tobacco smoke.

Material and methods: The study population consisted of 150 women from Lodz, Poland among which detail questionnaires were conducted three times during pregnancy period. From all women included into the study the saliva sample was collected to verify smoking status in pregnancy. One and two years after delivery additional questionnaires were performed and urine sample from all children was collected. The cotinine level in saliva and urine was analyzed using Liquid Chromatography with Tandem Mass Spectrometry (LC-MS/MS ESI+).

Results: About $22 \%$ of the children were expose to ETS during prenatal and $46 \%$ in postnatal period. Cotinine level in children's urine statistically differentiated children from smoking mothers (geometric mean (GM) $11.4 \mathrm{ng} / \mathrm{ml} ; 95 \% \mathrm{Cl} 7.1-18.3$ ) and in exposed homes (GM $7.3 \mathrm{ng} / \mathrm{ml}$; 95\% Cl 3.615.0) compared with non-exposed homes (GM 1.3 $\mathrm{ng} / \mathrm{ml} ; 95 \% \mathrm{Cl} 0.9-1.7)(p<0.001)$.

Conclusions: Children should be more protected from ETS exposure in prenatal and postnatal period.

1228

\section{ENVIRONMENTAL TOBACCO SMOKE EXPOSURE, PREGNANCY OUTCOMES AND CHILDREN'S HEALTH}

\section{K. Polanska}

Department of Environmental Epidemiology, Nofer Institute of Occupational Medicine, Lodz, Poland

Aim: The aim of the study was to analyze the influence of environmental tobacco smoke exposure on pregnancy outcome and children's health.

Material and methods: The study population consisted of pregnant women and their children followed up to two years of life. Pregnant women were interviewed three times during pregnancy.
From all women included into the study the saliva sample was collected. Assessment of child ETS exposure within two years after birth was based on questionnaires, confirmed by biochemical verification of cotinine level in child urine. Cotinine level in biological samples was analyzed using Liquid Chromatography with Tandem Mass Spectrometry. Pregnancy outcomes were noticed by neonatologist. The children's health focused on anthropometry indicators and the incidences of respiratory infections and allergy was performed by pediatricians during the first and second year of life.

Results: For the lower cotinine levels in saliva (up to $10 \mathrm{ng} / \mathrm{ml}$ ) we observed increasing trend in birth weight, head and chest circumference with the increasing level of cotinine ( $p$ trend $<0.05$ ). For the cotinine level higher than $10 \mathrm{ng} / \mathrm{ml}$ we noticed decreasing trend for birthweight, head and chest circumference as the level of this biomarker increased. The newborn length decreased with the increasing cotinine level in saliva ( $p$ trend $<0.001$ ). There were no statistically significant associations between ETS exposure and anthropometric measurements within first two years of life. Such exposure increases the risk of pneumonia but the results were not statistically significant.

Conclusions: All effort should be taken to eliminate the child ETS exposure.

1229

\section{GROWTH PATTERNS IN DUTCH CHILDREN WITH DOWN SYNDROME}

H.B.M. van Gameren-Oosterom ${ }^{1,2}$, P. Van Dommelen ${ }^{1}$, C.I. Lanting ${ }^{1}$, A.M. OudesluysMurphy $^{2}$, S. Buitendijk ${ }^{1}$, S. Van Buuren ${ }^{1}$, J.P. Van Wouwe ${ }^{1}$

${ }^{1}$ Netherlands Organisation for Applied Scientific Research, TNO, ${ }^{2}$ Pediatrics, Leiden University Medical Center, Leiden, The Netherlands

Objective: The majority of children with Down syndrome (DS) have concomitant disorders, such as congenital heart defects (CHD), celiac disease, and hypothyroidism. We aim to establish growth patterns of height in Dutch children with DS, while taking into account the influence of the concomitant disorders.

Methods: Out of the 30 regional outpatient clinics for children with DS in the Netherlands 25 participated. Children with Trisomy 21 karyotype of Dutch descent 